\title{
Covid-19 en la seguridad y salud en el trabajo, en un organismo público de Lima
}

\author{
Mtro. Lic. en Adm. Ernesto Gino Vilchez Luque \\ https://orcid.org/0000-0002-0340-7522 \\ Universidad César Vallejo \\ Lima - Perú
}

\section{RESUMEN}

El propósito del presente enfoque cualitativo fue, de analizar la COVID-19 en la Seguridad y Salud en el Trabajo-SST, en un organismo público de Lima y las medidas que tomaron para prevenir la proliferación de infectados de sus empleados y entorno familiar que, laboran en sus oficinas principales y anexos. Asimismo, la presente investigación se categorizó y subcategorizó apriorísticamente. Metodológicamente, se utilizó la investigación básica con el objetivo de explorar las vivencias de los principales protagonistas y también que sirva como aporte al mundo académico. Con relación al diseño, se basó en la fenomenología e historia de vida de los actores que narraron sus experiencias personales laborales y familiares, frente al Coronavirus y a través de entrevistas coordinadas, autorizadas previamente y respetando las disposiciones sanitarias nacionales e internacionales; como también utilizando las herramientas tecnológicas: el zoom, WhatsApp, correos electrónicos y las comunicaciones telefónicas. Que todo ello, respetando el rigor científico, los aspectos éticos, junto con la recolección, análisis de datos y los antecedentes encontrados, sirvió para la triangulación de la información, concluir, encontrar los resultados, discutir, concluir, recomendar y proponer mejoras de los hallazgos encontrados durante el proceso de la investigación efectuada.

Palabras clave: covid-19; seguridad y salud en el trabajo; triangulación; hallazgos. 


\title{
Covid-19 in occupational health and safety in a public organization in Lima
}

\begin{abstract}
The purpose of this qualitative approach was to analyze the COVID-19 in the Safety and Health at Work-SST, in a public agency in Lima and the measures taken to prevent the proliferation of infected employees and their families who work in their main offices and annexes. Likewise, this research was categorized and subcategorized aprioristically. Methodologically, basic research was used in order to explore the experiences of the main protagonists and also to serve as a contribution to the academic world. In relation to the design, it was based on the phenomenology and life history of the actors who narrated their personal work and family experiences, facing the Coronavirus and through coordinated interviews, previously authorized and respecting the national and international sanitary provisions; as well as using technological tools: zoom, WhatsApp, emails and telephone communications. That all this, respecting the scientific rigor, ethical aspects, together with the collection, data analysis and the background information found, served for the triangulation of information, conclude, find the results, discuss, conclude, recommend and propose improvements of the findings found during the process of the research carried out.
\end{abstract}

Keywords: covid-19; occupational safety and health; triangulation; findings.

Artículo recibido: 30 noviembre. 2021 Aceptado para publicación: 29 diciembre 2021 Correspondencia: pplanetariajusto@gmail.com Conflictos de Interés: Ninguna que declarar 


\section{INTRODUCCIÓN}

La COVID-19, por tratarse de un virus letal y pandémico, el modus vivendi del planeta dio un giro total de $360^{\circ}$, que no respetó la condición del status social, laboral, político, económico y religioso. Cambiando radicalmente el ritmo de sus vidas, enfrentando emocionalmente y tocando las fibras más íntimas ante la pérdida de sus seres queridos y optar por otras alternativas para subsistir, usando los medios tecnológicos para trabajar dependiente o independientemente o recibir la asistencia social por parte del Estado -en especial, a las personas vulnerables de salud y de precarios ingresos económicos- así cómo, el cumplimiento de las disposiciones emanadas tanto por el Gobierno Nacional y sus entes rectores, como de lo normado por los estamentos internacionales para acatar las medidas sanitarias de protección y confinamiento en sus hogares.

Por lo tanto, y no ajeno a lo descrito anteriormente, el presente trabajo de investigación -desde el punto de vista cualitativo-, tuvo como objetivo general describir la COVID-19 en la Seguridad y Salud en el Trabajo en un organismo público de Lima, la secuela causada por este flagelo mundial; así cómo, las medidas que adoptó la Alta Dirección para evitar el contagio y víctimas mortales -en lo mínimo-, y hasta que permanezca el virus en la población estudiada.

Con relación al COVID-19, se consultó el antecedente en el ámbito internacional, de la Organización Mundial de la Salud-OMS (2020), dónde su director -el Sr. Tedros Adhanom-, afirmó que la mejor defensa ante un brote pandémico, es un sistema sanitario consistente (haciendo historia de lo sucedido con el SIDA, sarampión, el Ébola, la tuberculosis y el paludismo), que obligadamente se tuvo que recurrir a la emergencia sanitaria mundial y así, detener su proliferación mortal.

El siguiente artículo, pertenece a la investigación de Valle (2020). Separando su artículo en dos partes; la primera, relacionando la génesis de este virus con otras pandemias; así como su implicancia en la sociedad, la economía global actual -y sin duda alguna- su afectación en el futuro, sin descartar la segunda ola de contagio que se presentará en cualquier momento. En tanto, en la segunda parte de su enfoque de investigación, analiza la toma de decisiones de diferentes gobiernos - en especial de España y Cantabria- con respecto a las políticas laborales de Estado, en beneficio de los trabajadores y empleadores; del mismo modo, de los efectos ante los posibles despidos, desempleos y de las pérdidas ocasionadas por este flagelo mundial. 
Castillo-Esparcia y Fernández-Souto et al. (2020). Analizaron el tema y manifiestan los efectos devastadores que ha provocado el coronavirus en toda su amplitud. Así como, la toma de decisiones que efectuaron los gobiernos para combatir este flagelo utilizando los diferentes medios de comunicación masiva para llegar a la población; y de esa manera reducir la curva de infección y víctimas mortales.

Larrea-Araujo et al. (2021). Precisan en su estudio realizado en el país ecuatoriano que, gracias a la toma de decisiones oportuna y preventiva de los gobiernos mundiales -en lo referente al aspecto laboral y trabajo remoto domiciliaria-, se evitó que la curva de crecimiento de infectados y cifras mortales se incrementaran. Por otro lado, se realizó un muestreo poblacional de Chi-Cuadrado y Fisher, a más de doscientos servidores, encontrándose problemas de salud con relación a la postura corporal y la no entrega de materiales de escritorio, con la finalidad de cumplir sus funciones a cabalidad. Recomendando, una mayor atención al personal en cuanto a salud se refiere y por otro lado, la entrega oportuna de muebles, equipos, teléfonos móviles e internet.

Por otro lado, el artículo particular de Chattopadhyay \& Pandit (2021). Manifestaron que, el objetivo del estudio fue de comprobar las diferencias en el trato y condiciones de ambiente laboral y remoto que reciben los trabajadores de los países en vías de desarrollo, por parte de los autores de esta pandemia pertenecientes a los países del primer mundo, quiénes pretender continuar avasallando a la población mundial.

La presente publicación, es con referencia a la SST y la Organización Internacional del Trabajo-OIT, quién su director -el Sr. Guy Rider afirmó-, para recuperarse sosteniblemente, la normatividad cumple un rol importante en las políticas de Estado a emplearse para tal fin. Promoviendo dignamente, el trabajo seguro, igualitario y con plena libertad a seguir. De igual forma, respetando sus principios laborales (más aún, ahora con esta pandemia), tomando acuerdos entre el gobierno, la clase trabajadora y las empresas. Así también -cómo lo enunciado anteriormente-, otro papel importante son las normas, en este caso referido a los estándares internacionales como es el Sistema de Gestión de Seguridad y Salud en el Trabajo-SGSST, obteniendo la publicación de Uhrenholdt et al. (2020), criticando al SGSST por no haber estimado seriamente a la normatividad ISO45001-2018 y a la OHSAS-18001; para tal afirmación, los resultados los halló a través de las teorías operacionales y otras que las integran, sirviendo como base para su implementación en las organizaciones. 
En el mismo sentido y en lo correspondiente a lo nacional, la plataforma del Estado peruano (2020) informó, haber realizado más de 1 millón 600 mil pruebas de descarte; asimismo, confirmó un total de 282,365 de casos positivos; de los cuales, 171,159 se recuperaron. Así también, cerca de 11 mil personas se hospitalizaron y 1,183 estuvieron en la Unidad de Cuidados Intensivos (UCI); también anunció, las más de 15 mil camas hospitalarias destinadas exclusivamente para Covid-19 y que pronto llegarán a 20 mil. Del mismo modo sostuvo que, al día hicieron un promedio de 15 mil pruebas de descarte e implementándose laboratorios para pruebas moleculares en 13 regiones del país. También, el gobierno de ese entonces anunció la conformación del Comando Vacuna (con la participación del sector privado, el Ministerio de Salud-MINSA, el Ministerio de Economía y Finanzas -MEF y el Ministerio de Relaciones Exteriores- MRREE, para asegurar el financiamiento, la obtención, la distribución y la aplicación de las vacunas.

De la misma manera -al inicio y en ese entonces-, se obtuvo el informe del ente rector de salud gubernamental, MINSA (2020). Anunciando, que los inicios de la enfermedad se originaron en China, en el 2018 y en Perú, en marzo del 2019, en que los síntomas y su transmisión provocan hasta la muerte de no tomarse las acciones que correspondan y para evitar su proliferación a nivel nacional.

En el mismo sentido, el ente rector laboral - La Autoridad Nacional del Servicio CivilSERVIR (2020). Informó que, ante la emergencia sanitaria, sin descuidar sus actividades laborales, tomando las medidas sanitarias de protección y otras disposiciones dictadas por el gobierno nacional, opten por las modalidades de trabajo, como es: el trabajo remoto, el presencial o semipresencial.

Asimismo, en lo que corresponde al ámbito local, el actual MIDAGRI, antes MINAGRI (2020). Desde el mes de marzo en que hizo su aparición este virus letal en el Perú y en que se suspendieron sus atenciones a la ciudadania de manera presencial, las autoridades adoptaron directivas de prevención para antes y al reincorporarse paulatinamente a sus actividades habituales.

Con relación, a uno de los objetivos específicos y subcategoría tomados en el trabajo es, los Factores de Riesgo Psicosocial Laboral-FRPL. Siendo el artículo publicado por van der Molen et al. (2020). Dónde estadísticamente y a través de un cuestionario elaborado prospectivamente para encuestar a más de 73,000 trabajadores europeos, sus resultados fueron la falta de interés por parte de los empleadores para respaldarlos en cuanto a la 
estabilidad laboral, emocional y protección asistencial. Asimismo, otro trabajo referido a los SG-SST es el de Dahler-Larsen et al. (2020), quiénes concluyen en la necesidad de considerar en las organizaciones públicas y europeas a los FRPL (lo humano), frente a otros aspectos administrativos de gestión.

Así también, tenemos otro de los objetivos específicos en esta categoría, como es el trabajo remoto. En que Irusta e Ingrassia (2020), en su estudio aplicado a las entidades públicas y privadas de Argentina puntualizan: quiénes ponen en funcionamiento la Tecnología de la Información y Comunicaciones-TICs en sus organizaciones, a la corta o a la larga obtendrán un mayor costo-beneficio, en comparación de quiénes no la ejecutan; por cuanto, lograrán un mayor control de sus actividades, como de su personal que labora remotamente.

Con relación al teletrabajo y al servicio social en Europa. Lavié et al. (2021). Afirman, el país más afectado por el Coronavirus fue España y que gracias a las medidas sanitarias empleadas aminoraron sus efectos colaterales adversos. Asimismo, para el presente estudio se tomó en cuenta el rol preponderante que cumplen los asistentes sociales en el país; para tal fin, centraron su estudio en entidades públicas de Madrid, cuyo resultado fue que la población se mostró con estrés y con malestar, ante la carencia de organización estatal y asistencia social en todo aspecto (nutricional, limpieza, difusión, ayuda psicológica, monetaria, entre otros), además de tener como alternativa de subsistencia, el trabajo remoto.

\section{ESTRATEGIAS METODOLÓGICAS}

Con respecto al tipo y diseño del presente trabajo de investigación fueron el básico y el fenomenológico-narrativo e historias de vidas, utilizando el instrumento de la entrevista. También, se tomó como muestra a la población que labora en sus diferentes locales, ubicados en los diferentes distritos limeños y tomando en cuenta el criterio de inclusión y exclusión de los entrevistados. Luego, de ubicar el ámbito temático, formular el problema y los objetivos, se procedió a categorizar y subcategorizar apriorísticamente. Siendo estas, la categoría de la SST, con su factor de riesgo psicosocial laboral, los estándares y normas y por último el trabajo remoto. Y la segunda categoría, el COVID19, con sus subcategorías: causa-efecto, las políticas de Gobierno, y por último, la normatividad laboral. Posteriormente, se elaboró la guía de entrevistas, con la finalidad de aplicar el instrumento, previo consentimiento informado y firmado por los 
especialistas en el tema. Luego de coordinar y proceder con las entrevistas presenciales adoptando las medidas sanitarias establecidas-, y también las remotas, utilizando las herramientas tecnológicas, como fueron: el zoom, el WhatsApp, el e-mail y las comunicaciones telefónicas; para luego, realizar el análisis de la triangulación con los datos recolectados y para su posterior obtención de resultados, discusión y conclusiones llegadas para tal fin.

\section{RESULTADOS Y DISCUSIÓN}

El resultado del análisis encontrado con relación al objetivo general de la SST, fue que la mayoría de los participantes mostraban similitud en sus opiniones en cuanto el organismo público decidiera que todos pasaran previamente por el control médico -para de esa forma-, disponer del personal que trabajaría presencial, semipresencial o remotamente, con el propósito de definir quiénes se encontraban en condiciones de laborar en algunas de las modalidades mencionadas y dispuestas por los organismos gubernamentales y rectores de salud y trabajo. Y el otro entrevistado agregó enfáticamente, que la atención y tratamiento médico -en el caso de ser víctimas del Coronavirus-, se amplie a los integrantes de sus hogares; por cuanto al infectarse uno o más, todos serían contagiados. Y en cuanto al segundo cuestionamiento, la respuesta resultó discordante; mientras que unos sostenían que la patronal les dotaba con los Elementos de Protección Personal-EPP y hasta la prueba rápida necesaria; otros reclamaban por, gastar de sus propios ingresos mensuales y afectar su economía familiar.

En lo referente, al análisis encontrado con relación al objetivo general del COVID-19 en la Seguridad y Salud en el Trabajo en un organismo público de Lima -al reiniciar sus labores-, los servidores adoptaron algunas de las modalidades emanadas por el órgano rector de salud y trabajo; así como, las medidas de prevención que tomó la entidad para evitar o reducir las víctimas de este flagelo. Para ello, el artículo de Akingbola (2020) concuerda con esta primera discusión, afirmando que los empleados al tener contacto permanente con el público, son los más proclives al contagio; por lo cual, el empleador tomará las medidas extremas y necesarias de protección; para llevar a cabo este resultado, el actor aplicó el sondeo y el muestreo no probabilístico en diversas ciudades de Europa. Así también, se obtuvo la publicación de Wiengarten et al. (2017) quién a través de su uso metodológico-analítico-explicativo- regresivo y el diseño Ancova-Mancova, concluyeron y compararon que los empleadores que adoptaron los estándares 
internacionales de SST, salieron beneficiados no solo quiénes componen la institución, sino también los usuarios y el ecosistema que los rodea.

En cuanto al primer objetivo específico correspondiente a conocer el COVID-19 en la SST, en un organismo público de Lima, con respecto al desarrollo de los Factores de Riesgo Psicosocial Laboral-FRPL. Por una parte, los trabajadores manifestaron su aprobación por la reciente contratación de especialistas en salud mental frente a la otra parte, al discrepar por la carencia de médicos ocupacionales y su deficiente atención, al existir sólo dos profesionales para la considerable cantidad de servidores que cuenta la institución pública. Pero si, aprobaron mayoritariamente la gestión de la Oficina General de Recursos Humanos-OGGRH, por la capacitación de diferentes tópicos relacionados con el COVID-19 y otros.

Es así que, conociendo el desarrollo de los factores de riesgo psicosocial laboral del COVID-19 y su influencia en la SST, en un organismo público de Lima. Los colaboradores, resaltaron la decisión de la Alta Dirección por contratar profesionales psicólogos, para afrontar emocionalmente la pandemia; mas no así y la necesidad también, de preocuparse para reclutar profesionales especializados en salud ocupacional y enfermeras, con el propósito de atender la demanda creciente de trabajadores y familias infectadas y con casos de muerte. Dando fe a lo señalado, Williams (2019) en su tesis doctoral cualitativa, aplicó entrevistas con especialistas del tema, para responder holísticamente acerca de este aspecto de vital importancia para la salud no sólo física, sino mental, salvaguardando el equilibrio y una vida llevadera.

Otro de los hallazgos asociados es, para explicar el objetivo específico del COVID-19 y su influencia en la SST en un organismo público de Lima, respecto a la contribución del trabajo remoto. Los especialistas mostraron su conformidad y con diferente criterio tomado por la Alta Dirección para continuar percibiendo su remuneración total y sin que les afecte su situación económica y familiar; al referir que, para clasificarlos en las modalidades de trabajo presencial, semipresencial o remoto, los consideró a unos por su condición física; a otros, por su estado psicosocial; y a otros, por sus años de vida.

Por lo cual, se trató de explicar la contribución del teletrabajo o trabajo remoto frente al COVID-19 y su influencia en la SST, en un organismo público de Lima. Los entrevistados especialistas, resaltaron el cumplimiento de la patronal, ante lo dispuesto por el gobierno nacional de turno, al evaluarlos por su condición física, edad y vulnerabilidad de salud, 
para de esa forma clasificarlos, quiénes trabajarán de manera remota, semipresencial o presencial y sin perjudicarlos económicamente en sus ingresos mensuales acostumbrados. Dando la razón, Valenzuela-García (2021) en su estudio seleccionando a España, por ser la ciudad representativa y la más perjudicada de Europa, al someter en un sondeo a cerca de setenta citadinos. Siendo la resultante de esta prueba, poco aprobatoria por considerarlo con demasiada carga laboral; sin embargo, otros se resignaron al no tener alternativa a seguir y como medio de subsistencia para enfrentar la crisis.

Con este penúltimo objetivo específico del COVID-19 en la SST en un organismo público de Lima, se estableció que el desarrollo de las Políticas de Gobierno reflejado en las entrevistas hechas a los servidores especialistas, tuvieron similar respuesta favorable. Aduciendo que, la gestión del gobierno de turno que enfrentó inicialmente, fue acertada y oportuna; así como los decretos de urgencia-DU publicados, para reducir el índice de contagiados y muertos.

Asimismo, los entrevistados tuvieron puntos de coincidencia al manifestar la acertada intervención del gobierno nacional en beneficio de la población laboral. Todo lo contrario, sucede con el artículo publicado por Vanegas y Jaramillo (2020), sosteniendo a pesar que el gobierno colombiano hizo denodados esfuerzos para paliar en todo orden la fuerte crisis provocada por el Coronavirus, el sector laboral es el que no ha sido tomado en cuenta; en razón, de estar despidiéndose a considerable cantidad de trabajadores.

En este último objetivo específico que, determina el COVID-19 en la SST en un organismo público de Lima, con relación al amparo y normatividad laboral. Uno de los trabajadores entrevistados, estuvo en desacuerdo con el incumplimiento de la patronal con ellos y los demás, sostuvieron que gracias tanto a las normas nacionales como internacionales, pudieron continuar con sus actividades personales y de estabilidad laboral, con absoluta normalidad. Y una, de las respuestas que más resaltó con relación al estar de acuerdo con el reclutamiento de nuevo personal para reforzar a los que trabajan remotamente -fue que, daría la razón-, pero la realidad es que se reclutó trabajadores, pero con una remuneración por encima de lo establecido.

Es así, se determinó la normatividad laboral existente, frente al COVID-19 en la SST, en un organismo público de Lima. Ante las respuestas brindadas por los entrevistados, al sentirse respaldados por los organismos nacionales e internacionales, para mantener su status laboral, económico y al ser pieza importante del engranaje del sostén familiar, 
desarrollo personal y laboral. El presente artículo de Occhi (2020), discrepa lo señalado. Al manifestar, que el trabajador en la República de Argentina, no se siente respaldado en sus derechos laborales, para enfrentar al COVID-19 y con el riesgo de perder su empleo.

\section{CONCLUSIONES O CONSIDERACIONES FINALES}

El objetivo general del desarrollo y normativo concluyó en su análisis, que el personal entrevistado no opinaba lo mismo, algunos afirmaban contar con el apoyo institucional para ellos y sus familias y otros no; siendo su origen, porque algunos laboran en las diferentes sedes y en sus diferentes modalidades: presencial, semipresencial y remoto. Por ejemplo, en el caso de las entregas de los elementos de protección personal-EPP y de las tomas de la prueba rápida y molecular. Es más, hasta algunos aducían que salían de sus bolsillos.

Con relación al primer objetivo específico, se dio a conocer el desarrollo de los Factores de Riesgo Psicosocial Laboral-FRSL. Afirmando los participantes, de contar con el incremento de profesionales en salud mental para paliar la situación emocional difícil que atraviesan por la cantidad de servidores infectados y fallecidos. Mas no así sucede, con los profesionales médicos ocupacionales y enfermeras que atienden deficientemente a la población considerable de trabajadores que cuenta la entidad, incluyendo sus familias. Teniendo en la actualidad que, apoyarse con personal administrativo voluntariado, quiénes acompañan -a los dos únicos médicos que existen-, en el monitoreo, la medicación y la renovación del oxígeno llevados a los domicilios de los trabajadores.

Otro objetivo específico, estuvo relacionado con el teletrabajo o trabajo remoto. En este caso, si estuvieron de acuerdo con las consideraciones especiales por la escogencia en su modalidad de trabajo -remoto-, siendo los más beneficiados los de cierta edad y salud vulnerable. Y con relación al pago de sus remuneraciones en su totalidad y otros más, que por convenio perciben vales alimenticios adicional a sus sueldos y en su condición de nombrados, reconocen lo dispuesto por la Alta Dirección y el cumplimiento a lo dispuesto por el gobierno nacional. Pero si, respecto a la entrega de útiles de escritorio, muebles, internet, telefonía y computadoras materiales y equipos de cómputo los entrevistados discrepan, a unos si les dotan y a otros les afecta económicamente. En síntesis -por el momento que se vive-, la alternativa del trabajo remoto y semipresencial benefició a los servidores públicos tanto para evitar el contagio del letal virus (para ellos y sus familias), 
como para continuar percibiendo su remuneración acostumbrada y sin riesgo al desempleo.

En el mismo sentido, el tercer objetivo específico, estableció el desarrollo de las Políticas de Gobierno. Resultante de las entrevistas realizadas, los participantes establecieron puntos de coincidencia, en cuanto a las acciones inmediatas que tomaron en conjunto, tanto del órgano rector de salud (MINSA) y el gobierno nacional (Política de Estado y de gobierno), con el propósito de minimizar los infectados y víctimas mortales, en especial de las personas con comorbilidad y con vulnerabilidad socio-económica.

Como última conclusión y tercer objetivo específico se determinó, la implementación y normatividad laboral. Aduciendo los entrevistados frente a las preguntas hechas, que sí recibieron el respaldo debido del organismo al cual pertenecen y el acatamiento de las normas gubernamentales establecidas, para conservar su estabilidad laboral y calidad de vida, Cuyas acciones se reflejaron en la contratación de personal adicional, con el fin de ejercer el respaldo de la labor presencial y ellos la remota, con la finalidad de protegerse del virus, tanto ellos, como de su entorno familiar. Pero también -por la otra parte de los entrevistados- se recibió la crítica, por cuanto afirmaron que las últimas contrataciones de servidores nuevos, fueron hechas con una paga mayor a lo que realmente se acostumbra y con una considerable diferencia que perciben ellos. Debiéndose, a la falta de un control concurrente o posterior.

\section{LISTA DE REFERENCIAS}

Akingbola, K. (2020). COVID-19: The Prospects for Nonprofit Human Resource Management. Canadian Journal of Nonprofit \& Social Economy Research / Revue Canadienne de Recherche Sur Les OSBL et l'économie Sociale, 11(1), 1620. https://doi.org/10.29173/cjnser.2020v11n1a372

Autoridad Nacional del Servicio Civil. Resolución de Presidencia Ejecutiva $N^{\circ} 0392020$ -

SERVIR-PE. 5 de junio de 2020.https://www.gob.pe/th/institucion/servir/normaslegales/920853-039-2020-servir-pe

2020.https://www.gob.pe/th/institucion/servir/normas-legales/920853-039-2020servir-pe

Castillo-Esparcia, Antonio; Fernández-Souto, Ana-Belén; Puentes-Rivera, Iván (2020). “Comunicación política y Covid-19. Estrategias del Gobierno de España”. 
Profesional de la información, v. 29, n. 4, e290419. https://doi.org/10.3145/epi.2020.jul.19

Chattopadhyay, S., \& Pandit, S. (2020). Freedom, distribution and work from home: Rereading engels in the time of the COVID-19-pandemic. Triple C, 19(1), 140153. https://doi.org/10.31269/triplec.v19i1.1225

Dahler-Larsen, P., Sundby, A., \& Boodhoo, A. (2020). Can occupational health and safety management systems address psychosocial risk factors? An empirical study. SafetyScience, 130,[104878]. https://doi.org/10.1016/j.ssci.2020.104878

Hidalgo Lavié, A., González Ramos, A. M., \& Lima Fernández, A. I. (2021). Social Work Practice during the COVID-19 State of Emergency in Spain. Social Work and Social Sciences Review, 22(2),88-102. https://doi.org/10.1921/swssr.v22i2.1585 file://C:/Users/Admin/Downloads/1585-Article\%20Text-3980-1-10$20210809 \% 20(2) \cdot p d f$

Irusta, J., \& Ingrassia, R. C. (2020). Estudio de caso: Entorno organizacional del trabajoremoto.http://rephip.unr.edu.ar/bitstream/handle/2133/19148/Irusta\%2C \%20Ingrassia Estudio\%

Larrea-Araujo, C.; Ayala-Granja, J.; Vinueza-Cabezas, A.; Acosta-Vargas, P. Ergonomic Risk Factors of Teleworking in Ecuador during the COVID-19. Pandemic: A Cross-Sectional Study. Int. J. Environ. Res. Public Health 2021, 18, 5063. https://doi.org/10.3390/ ijerph18105063

MINAGRI (2020). Lineamientos para el retorno progresivo al trabajo presencial en las entidades del Sector Agricultura y Riego. https://www.gob.pe/institución/minagri/normas-legales/609263-0070 2020minagri-sg

MINSA (2020). Conoce que es el Coronavirus. Resolucion-ministerial-039-2020MINSA.PDF.https://cdn.www.gob.pe/uploads/document/file/505245/resolucionministerial-039-2020-MINSA.PDF

Occhi, N. A. (2020). Los efectos de la terapia prescrita por el Derecho del Trabajo argentino contra el coronavirus COVID-19. 1-16. http://hdl.handle.net/2133/17974. 
Organización Internacional del Trabajo. (29 de mayo de 2020). Las Normas de la OIT y la COVID-19. https://www.ilo.org/global/standards/WCMS_739939/lang-es/index.htm

Organización Mundial de la Salud. (30 de marzo de 2020). La OMS publica directrices para ayudar a los países a mantener los servicios sanitarios esenciales durante la pandemia. http://www.who.int/es/news-room/detail/30-03-2020-who-releasesguidelenes-to-helps-countries-mantain-essential-health-services-duning-thecovid-19-pandemic13 (33), 120-128

Plataforma única del Estado peruano (30 de junio de 2020). Acciones del gobierno frente al COVID-19. Plataforma única del Estado peruano. Boletin. https://www.gob.pe/institucion/pcm/colecciones/793-anuncios-diarios-delgobierno-sobre-el-covid-19

Uhrenholdt Madsen, C., Kirkegaard, M., Dyreborg, J., \& Hasle, P. (september de 2020). Making occcupational health and safety management systems work: A realist review of the OHSAS 18001 standard. Scopus, 129. https.//www.scopus.com/record/display.uri?eid=2-s2.0$85085505841 \&$ origin=resultslist\&sort=plf

Valenzuela-García, H. (2021). Teletrabajo y Amplificación de la Desigualdad en la sociedad Post-Pandemia Española teleworking and the amplification of inequality in spains post-Pandemic Society. Revista Andaluza De Antropología, 1(19), 1436. https://doi.org/10.12795/RAA.2021.19.02

Valle, P. (2020). Impacto del Covid-19 en los Recursos Humanos y la administración de personal. Ucrea-Universidad de Cantabria. España. Recuperado. https://repositorio.unican.es/xmlui/handle/10902/19961.

van der Molen, HF, Nieuwenhuijsen, K., Frings-Dresen, M. y de Groene, G. (2020). Factores de riesgo psicosocial relacionados con el trabajo para los trastornos mentales relacionados con el estrés: una revisión sistemática actualizada y un metanálisis. BMJ abierto,10 (7), e034849. https://doi.org/10.1136/bmjopen$\underline{2019-034849}$

Vanegas Vásquez, C., \& Jaramillo Marín, R. S. (2020). El trabajo y la seguridad social en tiempos de la COVID-19. Opinión Jurídica, 19(40), 207-224. https://doi.org/10.22395/ojum.v19n40a10 
Wiengarten, F., Humphrys, P., Onofiei, G., \& Fynes, B. (january de 2017). The adoption of multiple certification standards perceived performance implications in quality, enviromental and health \& safety certifications. 28, 131-141. DOI: $10.1080 / 09537287.2016 .1239847$

Williams Jiménez, I. (07 de marzo de 2019). Riesgos Psicosociales emergentes y sus dimensiones reguladoras: una perspectiva internacional. http://hdl.handle.net/10016/28491 